\title{
A Contextual Foundation for Mechanics, Thermodynamics, and Evolution
}

\author{
Harrison Crecraft ${ }^{1, *}$ \\ 1 GeoEx Analytics: hcrecraft@geoexanalytics.com \\ * Correspondence: harrison@crecraft.net
}

\begin{abstract}
The prevailing interpretations of physics are based on deeply entrenched assumptions, rooted in classical mechanics. Logical implications include: the denial of entropy and irreversible change as fundamental properties of state; the inability to explain random quantum measurements and nonlocality without unjustifiable assumptions and untestable metaphysical implications; and the inability to explain or even define the evolution of complexity. The dissipative conceptual model (DCM) is based on empirically justified assumptions. It generalizes mechanics' definition of state by acknowledging the contextual relationship between a physical system and its positive-temperature ambient background, and it defines the DCM entropy as a fundamental contextual property of physical states. The irreversible production of entropy establishes the thermodynamic arrow of time and a system's process of dissipation as fundamental. The DCM defines a system's utilization by the measurable rate of internal work on its components and as an objective measure of stability for a dissipative process. The spontaneous transition of dissipative processes to higher utilization and stability defines two evolutionary paths. The evolution of life proceeded by both competition for resources and cooperation to evolve and sustain higher functional complexity. The DCM accommodates classical and quantum mechanics and thermodynamics as idealized non-contextual special cases.
\end{abstract}

Keywords: Physical Foundations; Quantum mechanics; Nonlocality; Time; Entropy; Thermodynamics; Origin of Life

\section{Introduction}

Physics has had a foundational crisis since the early Twentieth Century, when classical mechanics ceded its supremacy to quantum mechanics and relativity as fundamental descriptions of physics. Four fundamental problems highlight this crisis: 1) the problem of time, 2) the problem of contextuality of physical reality, 3) the problem of measurement, and 4) the problem of nonlocality.

\subsection{The Problems of Time}

Perhaps the most fundamental conceptual issue facing physics concerns the nature of time [1-4]. Relativity describes time as a dimension of spacetime, and like the three dimensions of space, time has no preferred direction. Change within spacetime is reversible and deterministic. Reversibility means that there is no fundamental arrow of time, and determinism means that the future is determined by the present. The future, as well as the past, is set in stone.

The fundamental determinism and reversibility of change is the logical consequence of what I refer to as the Hamiltonian conceptual model (HCM), which underlies classical mechanics, relativity, and many interpretations of quantum mechanics. The HCM defines the microstate, which expresses everything that is measurable and empirically knowable about a system, by perfect measurement in the absence of thermal noise. Perfect measurement in classical mechanics reveals (in principle) the precise positions and motions of a system's components and the forces acting on them. It follows from the HCM's definition of microstate and from the equations of motion that the classical mechanical microstate is deterministic.

Determinism by itself does not imply reversibility. Newton's three laws of mechanics do not include conservation of energy, and they accommodate irreversible dissipation of mechanical energy by frictional forces. 
The HCM goes beyond the empirical description of Newtonian classical mechanics by resolving a system into elementary particles. The HCM extrapolates the Newtonian mechanical state for macroscopic systems by defining the state by the precise coordinates and motions of elementary particles. Elementary particles have no internal parts. With no internal parts to vibrate or interact, a particle's internal energy equals the potential energy of its rest-mass, and its total energy therefore equals the sum of its particles' kinetic energy and potential energies. Kinetic and potential energies are quantified by their potential work, so the conservation of total energy means that work potential is conserved. This, along with determinism and perfect measurement, implies that we could, in principle, reverse the motions of particles and reverse the system's trajectory across time without external work. This is the definition of reversibility. Determinism and conservation of work potential together imply reversibility of the microstate. This applies to isolated systems for classical mechanics, quantum mechanics, and relativity

The HCM for classical mechanics assumes the microstate is a complete specification of the system's actual underlying physical state. The determinism and reversibility of the microstate means that the physical state, as it exists in isolation and unperturbed, evolves deterministically and reversibly.

Reversibility does not imply equal probabilities for time's directions, however. Physics acknowledges time's empirical asymmetry and the thermodynamic arrow of time, as recorded by the irreversible increase in entropy. Boltzmann defined entropy by a system's disorder, which he related to the number of accessible microstates consistent with the system's thermodynamic macrostate description. The macrostate is defined by a system's coarse-grained measurements due to thermal noise. Boltzmann's entropy is a measure of uncertainty of the system's actual physical state due to imperfect measurement. The irreversible increase in entropy reflects the statistical tendency for large numbers of initially ordered particles to disperse and become increasingly disordered and uncertain, and it defines an empirical arrow of time. The HCM, however, regards entropy and its increase as an emergent property of a macrostate and its imperfect description, and not as a fundamental property of the microstate or physical state.

In his book, The End of Certainty [5] (see also [6]), Ilya Prigogine sought to extend classical and quantum mechanics to accommodate irreversibility and the thermodynamic arrow of time as fundamental. He asserted that statistical descriptions for both classical and quantum systems are necessitated by two key factors: instabilities and "persistent interactions." He attributed instabilities to Poincare resonances and persistent interactions to sustained environmental fluctuations. Prigogine argued that these statistical extensions mark the end of certainty and establish irreversibility and randomness as fundamental properties. However, he implicitly assumed, without justification, that environmental fluctuations are fundamentally random. Within the prevailing interpretations of classical and quantum mechanics, however, environmental fluctuations are deterministic, and Prigogine's statistical formulations for irreversibility describe emergent, not fundamental, realities.

The thermodynamic arrow of time is not the only empirical arrow of time. The universe has evolved from a nearly homogeneous state following the Big Bang to its current state of extraordinary diversity and complexity. In our own corner of the universe, prebiotic chemicals evolved into self-replicating life forms, as early as 0.1 billion years after formation of the Earth's oceans [7]. Diverse ecosystems have subsequently spread throughout the oceans and across the globe. Much more recently, humans have evolved culture, technology, and a global economy. These trends exemplify the empirical arrow of evolving complexity.

The determinism of the HCM implies that the existing complexity of the universe must have been encoded in the initial state of the universe. The origin of such a finely tuned initial state for our universe, however, defies physical explanation. There is not even a measure for the internal organization that we associate with complex systems. The failure to account for the universe's state of extraordinary complexity has motivated interest in the idea of a multiverse and the anthropic principle, in which our universe is a rare statistical outlier that has allowed us to exist and ponder its origin [8]. 
With the HCM's reversibility and determinism of the physical state, there is no fundamental arrow of time, and there is no irreversible dissipation or evolution of complexity. There just is. Reality is a static block in $4 \mathrm{D}$ spacetime spanning the past, present and future. This is the block model of the universe, and it expresses the doctrine of Eternalism $[2,9,10]$.

\subsection{The Problem of Contextuality}

The microstate and physical state in classical mechanics and relativity are independent of an observer's inertial reference frame, aside from an information-preserving Galilean or Lorentz transformation. This means that there is no preferred observer or inertial measurement framework. The physical state for classical mechanics and relativity can therefore be defined independent of the physical state and as non-contextual.

With the discovery of quantum phenomena in the early twentieth century, it became clear that the laws of classical mechanics break down for very small particles, and a new theory was needed. Quantum mechanics defines the quantum microstate prior to measurement by the Schrödinger wavefunction:

$$
\Psi=\sum c_{i} \psi_{i} .
$$

The $c_{i}$ 's are complex weighting factors for the measurable eigenstates, $\psi_{i}$. The wavefunction is defined over time by a continuous function, and its change is therefore deterministic. Following classical mechanics, the HCM takes the microstate as a complete specification of the physical state, so the physical quantum state, as it exists isolated, unperturbed and unobserved, is also taken to be deterministic.

Individual quantum measurement results, however, are statistical. For example, repeated measurements of identically polarized photons reveal a statistical mix of photons, having polarization either parallel to or perpendicular to the measurement device's polarizer. Quantum mechanics preserves the determinism of the pre-measurement quantum state by defining perfect and complete measurement by quantum-state tomography [11]. This uses statistical measurement results on an ensemble of identically prepared systems and the Born rule to reconstruct the system's microstate as it existed prior to measurement.

As proven by the Kochen-Specker theorem [12] and as just described for measurements of photons' polarization, individual measurements are contextually dependent on the measurement framework. The superposed wavefunction describing a system's state, as it exists in isolation prior to measurement, however, is independent of the measurement framework. The HCM thereby preserves the non-contextuality of the pre-measurement quantum mechanical microstate and physical reality. The costs of non-contextuality are the problems of time, measurement, and non-locality.

Not all interpretations of quantum mechanics adhere to the HCM assumptions. The consistent histories interpretation [13], for example, asserts that the physical state is defined by eigenstates, which are contextually defined by the system's measurement framework. In Quantum Bayesianism [14], the state is contextually defined and updated by an observer's information. Contextual interpretations are motivated by efforts to resolve the outstanding conceptual problems of quantum mechanics, but they typically come at the cost of abandoning the objectivity of physical reality.

\subsection{The Problem of Measurement}

Observation of measurement results for an ensemble of identically prepared radioactive particles, subsequent to their preparation, reveals a statistical mix of decayed and undecayed microstates. If the superposed wavefunction is just an empirical description of the system's physical state, then the collapse from a pure superposed microstate to a mixed macrostate simply reflects a change in the system's description, based on new information acquired at observation.

However, as described in section 1.2, the HCM regards the wavefunction as a complete specification of the underlying physical state. The determinism and completeness of the wavefunction description implies that as long as a system remains isolated from external 
perturbations or observation, there can be no randomness. This, in turn, implies that the random collapse of a pure wavefunction to a statistical mix of eigenstates occurs when the system's isolation is violated, as it must be when we observe a system or its measurement results.

Erwin Schrödinger tried to highlight the absurdity of equating a superposed wavefunction with a system's physical state. He considered a perfectly isolated system comprising a radioisotope and its measurement apparatus. For added drama, Schrödinger used a cat for the measurement device. If the particle decays, the cat dies. At observation, we invariably find the cat either dead, indicating that the particle decayed, or alive, indicating no decay. Equating the physical state with the superposed and deterministic microstate implies that, while isolated and prior to its observation, the radioisotope and cat exist as a physically superposed state of undecayed-decayed and live-dead. Schrödinger rejected the wavefunction's physical reality and the superposition of physical states, and he proposed his experiment to illustrate the absurdity of its implications.

Hugh Everett proposed an alternative HCM interpretation that avoids the absurdity of superposed cats. In essence, his Many Worlds Interpretation [15] says that everything that can happen does happen in separate branches of an exponentially branching universe. Even we, as observers, are split. Each of our split selves observes our own particular branch and sees only a single outcome. We perceive random wavefunction collapse, but from the objective perspective of the universe as a whole, there is no random selection and the universe evolves deterministically. The Many Worlds Interpretation trades the absurdity of superposed cats for an exponentially branching universe instead.

Both of these interpretations are consistent with observations, and despite their untestable metaphysical implications, they ranked high in a survey at a foundations of quantum mechanics conference [16]. The measurement problem and the role of the observer on triggering the apparent randomness of observed results nevertheless remain unresolved conceptual problems of quantum mechanics [17].

\subsection{The Problem of Nonlocality}

A closely related and unresolved issue is the violation of locality by quantum mechanics [18]. Locality asserts that effects cannot propagate superluminally from a cause. Numerous Bell-type experiments indicate that nonlocality is not just a property of the quantum state description, but that it is a feature of a system's physical state $[19,20]$. Experiments show that measurements on spatially separated but entangled particle pairs are correlated, even if measurements are spatially separated and simultaneous. Nonlocality cannot be used to transmit signals superluminally, and there is no empirical conflict with relativity. However, there is no satisfactory explanation of how quantum nonlocality and relativity can coexist, and this poses a significant and unresolved conceptual problem $[18,21]$.

\subsection{We Need a Better Conceptual Model}

The nature of a system's physical state, while it is isolated and unobserved, cannot be resolved experimentally. It is strictly a matter of the assumptions on which experimental results are interpreted. The implications of HCM interpretations, however, are problematic-eternalism, the measurement problem, non-locality, and the exquisite fine tuning needed to simultaneously explain the empirical arrows of both increasing entropy and increasing complexity.

Any viable interpretation of quantum mechanics necessarily makes predictions consistent with observations. This begs the question of what difference any particular interpretation really makes? There has been a strong sentiment among prominent physicists to dismiss the philosophy of science. Richard Feynman is credited with saying: "The philosophy of science is as useful to scientists as ornithology is to birds." Efforts to understand what quantum mechanics means are countered the edict: "Shut up and calculate!" [22].

We take a different position. Seeking an objective interpretation of physical reality is more than an idle intellectual exercise, and it has real-world consequences. The universe is not a static block in spacetime, unchanging for eternity. Recognizing the objective reality of irreversible dissipative 
processes and explaining their behavior in terms of fundamental physical principles is essential if we want to understand how nature works. To advance physics beyond its current focus on states, we need a conceptual model that embraces irreversible dissipative processes and the spontaneous self-organization of dissipative systems.

\section{The Dissipative Conceptual Model of State}

The dissipative conceptual model (DCM), like any conceptual model, is an axiomatic system. An axiomatic system starts with simple assumptions that we accept as true. A conceptual model accepts empirical laws of Nature as true, and it seeks to explain observations in terms of its interpretation of physical reality.

None of the prevailing interpretations of quantum reality explains time's arrows, the measurement problem or nonlocality, while also maintaining an objective physical reality. This is a direct consequence of the Hamiltonian conceptual model's assumptions. The DCM takes a very different approach. Whereas the HCM is based on the idealizations of mechanics and interprets thermodynamics and its Second Law as a statistical approximation of mechanics, the DCM accepts the well-documented and universal principles of thermodynamics as fundamental truths.

\subsection{The Postulates of State}

The DCM's interpretation of physical state is based on the following postulates and definitions:

Postulate 1: No system has surroundings at absolute zero temperature and no system can be perfectly insulated from its ambient surroundings.

Definition 1: A system's ground state is defined by thermodynamic equilibrium with the system's ambient surroundings.

Definition 2: Perfect measurement is a reversible transformation from a system's initial state to its ground state.

Postulate 2: At perfect measurement, there are no hidden variables. Perfect reversible measurement therefore provides complete information on the system's physical state.

Postulate 3 ( $1^{\text {st }}$ Law of Thermodynamics): The total energy for a system plus its surroundings is conserved. A system's energy is conserved in the limit of perfect isolation.

Postulate 4 (Generalized $2^{\text {nd }}$ Law of Thermodynamics): Any irreversible transition between states is associated with the production of entropy.

Postulate 1 says that absolute zero temperature can be approached, but it is an unattainable idealization. No system is perfectly isolated from its surroundings and all systems exist and interact with their surroundings at a positive ambient temperature. The universe, by definition, has no surroundings, but its ambient cosmic background temperature at 2.7 kelvin permeates the vacuum of space, and this defines its ambient temperature. Postulate 1 provides the basis for the contextual interpretation of a physical system as it exists with respect to its ambient surroundings.

Definitions 1 and 2 define perfect measurement by a reversible transformation between a system and its ground-state reference (Figure 1). A perfect ambient observer is defined as an agent or device that can reversibly record the system's changes during this transformation. By recording the ambient transformation process and then reversing it, the perfect ambient observer can reverse the process and restore the system to its initial state. We generally consider perfect measurement to include both measurement and its reversal. In the limit of perfect reversible measurement and reversal, the system's initial state is left undisturbed. 


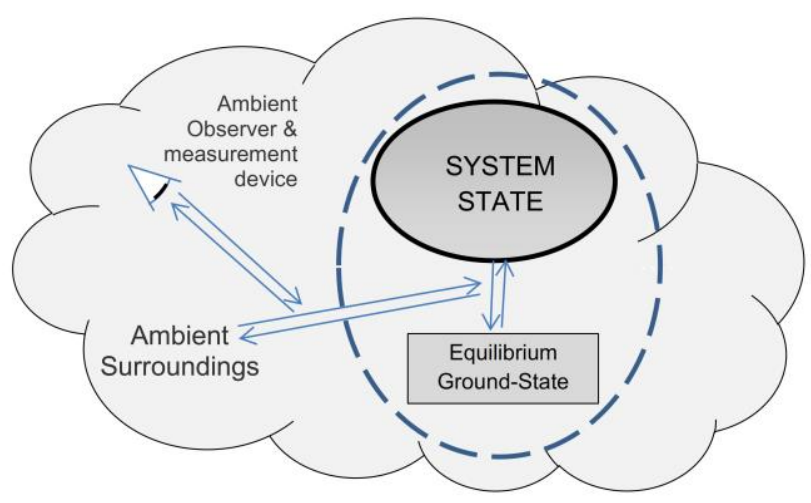

Figure 1. Dissipative Conceptual Model of State. The DCM defines a system's state relative to its equilibrium ground state and within the context of its ambient surroundings. Perfect measurement is an equilibrium transformation from the system's state to its equilibrium ground-state reference and reversible interaction with its ambient surroundings. Perfect measurement is reversible, with or without an observer, but actual reversal requires an ambient observer or measurement device to record and then reverse the process.

Perfect reversible measurement is necessary for the definition of a microstate, but reversible measurement is not always possible. The Quantum Zeno effect [23] shows that a continuously measured (and measurable) state does not change irreversibly. The contrapositive of this is equally true: an irreversibly changing system is not continuously (or reversibly) measurable. A system can be in a measurable and reversible state between irreversible transitions, but during transition it is not reversibly measurable, and it therefore does not exist as a DCM state.

The DCM partitions a system's total energy into energy components, given by:

$$
\mathrm{E}=\mathrm{Qgs}_{\mathrm{gs}}+\mathrm{E}_{\mathrm{sys}}=\mathrm{X}+\mathrm{Q}+\mathrm{Qgs}_{\mathrm{gs}}
$$

Ground-state energy, $\mathrm{Qgs}_{\mathrm{gs}}$ is the positive energy of the ambient ground state reference. Any excess energy is the system energy, $E_{\text {sys. }}$ The DCM resolves system energy into exergy $(X)$ and ambient heat (Q). Exergy is defined by the potential work that can be done on the ambient surroundings. Ambient heat is heat at the ambient temperature, and it has zero potential for work on the ambient surroundings. The total energy and ground-state energy are defined relative to absolute zero, but the system energy, ambient heat, and exergy are contextually defined relative to the ambient ground state, which sets their zero values.

Exergy includes all potential and kinetic energy, and a fraction of the thermal energy, given by

$$
d X_{q} \equiv \frac{\left(T-T_{a}\right)}{T} d q
$$

$\mathrm{dX}_{\mathrm{q}}$ is an increment of thermal exergy and $\mathrm{dq}$ is an increment of thermal energy equilibrated at temperature $\mathrm{T}$. The system temperature $\mathrm{T}$ is generally (but not necessarily) equal to or greater than the ambient temperature $T_{a}$. The remainder of thermal energy increment is ambient heat, given by

$$
d Q \equiv\left(T_{a} / T\right) d q
$$

Postulate 2 is a statement about the DCM's conceptual model of physical reality. Postulate 2 contextually defines physical reality by perfect measurement from a system's actual ambient surroundings. Perfect ambient measurements of a gas's particles' positions, for example, can only yield a probability distribution. The probability distribution is not statistical, as this would imply an incomplete specification of the particles' "actual" positions. The probability distribution is a complete specification of particles' positions for the gas as it exists in the context of its ambient surroundings. Probabilities refer to the potential actualization of more highly refined positions, such as by measurement at a new and much colder ambient temperature. 
A state, as it exists in context of its actual ambient surroundings, has no hidden properties, so properties beyond what are measurable from the ambient surroundings do not exist. The microstate is a complete description of the physical ontological state. "State," without qualification, will refer to both the microstate and the physical state.

Postulate 3 is a restatement of thermodynamics' First Law, expressing the conservation of energy for a system and its surroundings. Conservation of total energy and equation (2) imply that, in the limit of perfect isolation,

$$
\Delta \mathrm{E}=\Delta \mathrm{Q}+\Delta \mathrm{X}+\Delta \mathrm{Qgs}_{\mathrm{s}}=0,
$$

where the deltas refer to changes over time.

If the ambient surroundings are fixed, the ambient ground-state energy is likewise fixed, and equation (4) expresses the conservation of energy during irreversible dissipation of exergy to ambient heat. Equation (4) also expresses conservation of energy during changes in the ambient surroundings. A change in the ambient surroundings changes the ground-state energy and redistributes the system energy, but in the limit of perfect isolation, a system's total energy does not change.

Postulate 4 is a generalization of thermodynamics' Second Law. It states that any irreversible transition produces entropy and increases the total entropy of the system and its surroundings. Thermodynamics defines entropy for a closed system ${ }^{1}$ by:

$$
d S=\frac{d q}{T}
$$

where dS is the incremental increase in entropy due to addition of an increment of thermal energy $\mathrm{dq}$ at temperature T. The DCM contextually defines entropy with respect to the ambient temperature by:

$$
S_{D C M} \equiv \int_{T_{a}}^{T_{s y s}} \frac{d q}{T}=\frac{Q}{T_{a}} .
$$

The last term follows from integrating equation $3 b$ from $T_{a}$ to $T_{\text {sys. }}$ As the ambient temperature approaches absolute zero, the SDCM approaches thermodynamics' Third Law entropy, $\int_{0}^{T_{s y s}} \frac{d q}{T}$, which is equal to the statistical mechanical entropy. Sрсм is a contextually defined generalization of the statistical mechanical entropy for $T_{a}$ not equal to absolute zero.

The DCM entropy is a function of three independent variables: system temperature, ambient temperature, and a reaction progress variable, zeta $(\zeta)$, which indexes isothermal changes in state. By integrating partial differentials with respect to each of these variables and grouping, the DCM

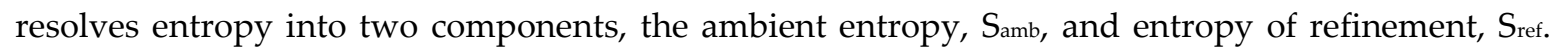
These are defined in equation 7 and illustrated in Figure 2:

$$
\begin{gathered}
S_{D C M}=\left(S_{a m b}\right)+\left(S_{r e f}\right) \\
\equiv\left(\int_{0}^{1}\left(\frac{\partial q}{\partial \zeta}\right)_{v, T_{s y s}, T_{a}} \frac{d \zeta}{T_{a}}+\int_{T_{a}}^{T_{s y s}} C_{v}\left(T_{s y s}\right) \frac{d T_{s y s}}{T_{s y s}}\right)+\left(\int_{T_{a, 0}}^{T_{a}}-C_{v}\left(T_{a}\right) \frac{d T_{a}}{T_{a}}\right) \\
=\left(S_{H C M, a s s}-S_{H C M, a g s}\right)+\left(S_{H C M, s y s}-S_{H C M, a s s}\right),
\end{gathered}
$$

where $\mathrm{C}_{\mathrm{v}}$ is the volumetric heat capacity, $\left(\frac{\partial q}{\partial T}\right)_{v, \zeta}$. S $_{\mathrm{HCM}, \mathrm{sys},} \mathrm{S}_{\mathrm{HCM}, \text { ass }}$ and $\mathrm{SHCM}_{\mathrm{H}, \mathrm{ags}}$ are entropies for the system state at its system temperature, the ambient system state, and ground-state reference (Figure 2). The HCM entropies are equal to the statistical mechanical entropy and the $3^{\text {rd }}$ Law thermodynamic entropy.

\footnotetext{
1 A closed system is closed to exchange of mass but open to exchange of energy
} 


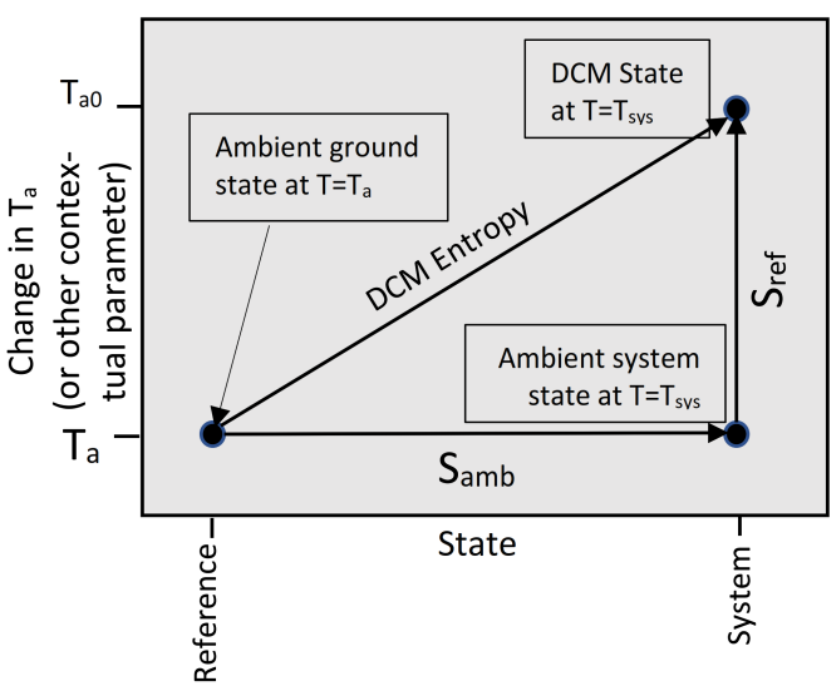

Figure 2. The Two Components of Entropy. The DCM resolves entropy into the entropy of refinement (Sref) and ambient entropy (Samb).

The entropy of thermal refinement (Sref, vertical vector in Figure 2) is a consequence of a change in the ambient reference, but before the system readjusts to the change. Only contextual properties, such as entropy and exergy, are changed by a change int the ambient surroundings; the system's temperature and total energy are unaffected. Equation 7 defines the entropy of refinement by integrating the partial derivative from the system's initial ambient temperature to its changed ambient temperature. It is positive for $\mathrm{T}_{a}<\mathrm{T}_{\mathrm{a}, 0}$.

The ambient entropy (Samb, horizontal vector in Figure 2) reflects change from the ambient ground state (defining zero entropy) to the ambient system state at fixed ambient temperature. Equation 7 resolves the change into an isothermal reaction, progressing from $\zeta=0$ to $\zeta=1$, and a direct exchange of heat between the system and ambient surroundings, with system temperature progressing from $\mathrm{T}_{\mathrm{a}}$ to $\mathrm{T}_{\text {sys. }}$.

The DCM also recognizes two distinct paths for entropy production. The first path of entropy production is the path of dissipation:

$$
\Delta S_{\mathrm{amb}}=-\Delta \mathrm{X} / \mathrm{T}_{\mathrm{a}}
$$

where $\Delta S_{\text {amb }}$ is the production of entropy due to dissipation of exergy, $-\Delta X$, at a fixed ambient temperature, $T_{a}$.

The second path of increasing entropy is by refinement associated with a change in ambient surroundings. Differentiating equation 7 with respect to $T_{a}$ at fixed $T_{\text {sys }}$ shows that the increase in the entropy of thermal refinement from a decline in ambient temperature is given by:

$$
\frac{\mathrm{dS}_{\mathrm{ref}}}{d T_{a}}=\frac{-\mathrm{C}_{\mathrm{v}}}{T_{a}} .
$$

Dissipation at a fixed ambient temperature (equation 8) and refinement, commonly associated with a declining ambient temperature (equation 9), describe two distinct paths leading to the irreversible production of entropy and the thermodynamic arrow of time.

\subsection{The Dissipative Classical State}

To illustrate the dissipative classical state, we consider an ideal classical monatomic gas prepared at equilibrium with ambient surroundings at 500K. Measurement of the gas's temperature, pressure, and volume determines its total energy and defines its thermodynamic macrostate (Table 1). The thermodynamic macrostate is non-contextual, and it does not depend on the ambient temperature. The gas's DCM microstate is contextually defined by the thermodynamic macrostate plus the system's contextually defined DCM entropy and energy components, listed in Table 1. 
Table 1. DCM Macrostates and Contextual Microstate Properties

\begin{tabular}{|c|c|c|c|}
\hline $\begin{array}{l}\text { Energy } \\
\text { Component }\end{array}$ & $\begin{array}{l}\text { n-Particle } \\
\text { Ideal Gas }\end{array}$ & Hydrogen Atom at Temperature $\mathrm{T}$ & $\begin{array}{l}\text { Contex- } \\
\text { tual? }{ }^{(1)}\end{array}$ \\
\hline $\begin{array}{l}\text { Macrostate } \\
\text { Description }\end{array}$ & $\begin{array}{c}\text { Temperature, Pressure, } \\
\text { Volume, Mass (n particles) }\end{array}$ & $\Psi(T)=\sum_{i} c_{i}(T) \psi_{i}$ where $\sum_{i}\left|c_{i}(T)\right|^{2}=1$ & No \\
\hline Energy (total) & $\mathrm{E}=\mathrm{nk}_{\mathrm{B}} \mathrm{T}$ & $\langle\mathrm{E}(\mathrm{T})\rangle=\sum_{\mathrm{i}} \mathrm{E}_{\mathrm{i}} \times\left|c_{\mathrm{i}}(\mathrm{T})\right|^{2}$ & No \\
\hline $\begin{array}{l}\text { Qgs (ground-state } \\
\text { energy) }\end{array}$ & $\mathrm{Qgs}_{\mathrm{g}}=\mathrm{nk}_{\mathrm{B}} \mathrm{T}_{\mathrm{a}}$ & $\mathrm{Q}_{\mathrm{gs}}=\mathrm{E}\left(\mathrm{T}_{\mathrm{a}}\right)=\sum_{i} E_{i} \times\left|c_{i}\left(\mathrm{~T}_{a}\right)\right|^{2}$ & Yes \\
\hline Esys (system energy) & $\mathrm{E}_{\mathrm{sys}}=\mathrm{nk}_{\mathrm{B}}\left(\mathrm{T}-\mathrm{T}_{\mathrm{a}}\right)$ & $\left\langle\mathrm{E}_{\mathrm{sys}}(\mathrm{T})\right\rangle=\langle\mathrm{E}(\mathrm{T})\rangle-\mathrm{Q}_{g s}=\sum_{i} E_{i}\left(\left|c_{i}(T)\right|^{2}-\left|c_{i}\left(T_{a}\right)\right|^{2}\right)$ & Yes \\
\hline $\begin{array}{l}\mathrm{Q}=\mathrm{T}_{\mathrm{a}}\left(\mathrm{S}_{\mathrm{ref}}+\mathrm{S}_{\mathrm{amb}}\right) \\
\text { (ambient heat) }\end{array}$ & $\mathrm{Q}=T_{a}\left(\int_{T_{a}}^{T_{s y s}} C_{v} \frac{d T}{T}+0^{(2)}\right)$ & $\langle\mathrm{Q}(\mathrm{T})\rangle=T_{a}\left(\int_{T_{a}}^{T_{s y s}}\left(\frac{\partial\left\langle\mathrm{E}_{\mathrm{sys}}(\mathrm{T})\right\rangle}{\partial T}\right) \frac{d T}{T}+0^{(2)}\right)$ & Yes \\
\hline X (exergy) & $\mathrm{X}=\mathrm{E}_{\mathrm{sys}}-\mathrm{Q}$ & $\langle X(T)\rangle=\left\langle E_{\text {sys }}(T)\right\rangle-\langle Q(\mathrm{~T})\rangle$ & Yes \\
\hline $\begin{array}{l}\mathrm{SDCM}=\mathrm{Q} / \mathrm{T}_{\mathrm{a}} \\
\text { (entropy) }\end{array}$ & $\mathrm{S}_{D C M}=\frac{Q}{T_{a}}=\int_{T_{a}}^{T_{s y s}} C_{v} \frac{d T}{T}$ & $\left\langle S_{D C M}(\mathrm{~T})\right\rangle=\frac{\langle\mathrm{Q}(\mathrm{T})\rangle}{T_{a}}=\int_{T_{a}}^{T_{\text {sys }}}\left(\frac{\partial\left\langle\mathrm{E}_{\mathrm{sys}}(\mathrm{T})\right\rangle}{\partial T}\right) \frac{d T}{T}$ & Yes \\
\hline
\end{tabular}

$\mathrm{k}_{\mathrm{B}}=$ Boltzmann constant. $\mathrm{C}_{\mathrm{v}}=$ volumetric heat capacity. 〈Angle brackets〉 indicate the time-averaged property for a superposed macrostate at temperature $\mathrm{T}>\mathrm{T}_{\mathrm{a}}$. $\mathrm{E}_{\mathrm{i}}$ is the total energy of the quantum mechanical eigenstate $\psi_{i}$.

(1) Contextuality only considers ambient temperature, not relative velocity, which would affect kinetic energy.

(2) Cooling to the ambient temperature brings the system to its ambient ground state, so $S_{a m b}=0$ (see Figure 2).

If we insulate the gas and lower its ambient temperature to $300 \mathrm{~K}$, its exergy increases. The gas's state is metastable; it has a potential to spontaneously approach its new ambient ground state at $300 \mathrm{~K}$, but the process is suspended by the insulation. Simply reducing the system's ambient temperature immediately changes the gas's contextual properties, as shown in Table 1.

Perfect reversible measurement of the metastable gas from the new ambient temperature involves extracting energy (e.g. by employing a reversible heat engine) until the gas reaches $300 \mathrm{~K}$. The process exchanges entropy between the gas and its ambient surroundings, but it is reversible, and no entropy is produced. Reversing the measurement process uses the extracted exergy to pump ambient heat back, restoring the gas's $500 \mathrm{~K}$ state as it contextually exists at the $300 \mathrm{~K}$ ambient temperature.

\subsection{The Dissipative Quantum State}

To illustrate the quantum dissipative state, we switch from describing a gas of essentially inert particles to describing the particles' internal states as they interact with ambient (black body) photons. We consider an ensemble of hydrogen atoms prepared in equilibrium with ambient photons at $6000 \mathrm{~K}$. The ensemble is below the temperature range for ionization of hydrogen (7,000-10,000K), but high enough that multiple energy levels are occupied, so the atom's energy is described as a superposed wavefunction.

Quantum mechanics describes the hydrogen's quantum state, $\Psi$, based on statistical measurements of transitions between the atoms' discrete energy levels. A spectrometer sufficiently close to absolute zero ${ }^{2}$ could in principle measure the emitted photons with high precision and determine the statistical distribution of energy transitions.

2 A hydrogen atom at $300 \mathrm{~K}$ is essentially in its ground-state energy, so $300 \mathrm{~K}$ is sufficiently close for high but finite precision. 
Measurements would reveal a statistical distribution of discrete and measurable eigenstates, $\psi_{i}$, as expressed by the ensemble's superposed wavefunction (Table 1). Individual eigenstate energies are quantized and independent of temperature. The $c_{i}$ weighting coefficients do depend on the ensemble's temperature of equilibration, however. The superposed wavefunction's energy consequently depends on temperature, but it is independent of the ambient temperature and it is therefore non-contextual.

The HCM interprets the superposed wavefunction as a microstate and a complete description of the physical state. In contrast, the DCM interprets it as a macrostate and an incomplete description of the system's instantaneous physical state, as it randomly fluctuates amongst its allowable microstates. The macrostate's average energy is $\langle E(T)\rangle=\sum_{i}\left|c_{i}(T)\right|^{2} E_{i}$, where $E_{i}$ is the energy of eigenstate $\psi_{i}$, and $\left|c_{i}(T)\right|^{2}$ is the probability that a hydrogen atom exists in the measurable eigenstate $\psi_{i}$ at any given instant (independent of actual measurement). The DCM microstate at any instant is completely defined by the wavefunction macrostate together with the instantaneous contextual properties listed and defined in Table 1.

A superposed wavefunction is generally an incomplete macrostate description of the physical state. However, for an ambient temperature of $6000 \mathrm{~K}$, the hydrogen atom at $6000 \mathrm{~K}$ is in its ambient ground state. The superposed wavefunction for a system's ground state completely describes its zero-entropy and zero-exergy microstate and physical state.

\subsection{Entropy, Refinement, and Measurement}

The HCM entropy for a quantum system is the von Neumann entropy [24], which can be expressed by:

$$
S_{v N}=-\sum_{i} \eta_{i} \log \left(\eta_{i}\right)
$$

The $\eta_{i}$ are the statistical probabilities of measurable eigenstates, as described for a mixed state following the collapse of a pure (zero-entropy) superposed quantum state. If the probability $\eta_{i}$ of the eigenstate $i$ is known to equal one and the remaining probabilities are zero, there is no uncertainty, and the system's $S_{\mathrm{vN}}$ equals zero. Uncertainty and $\mathrm{S}_{\mathrm{vN}}$ are maximized when all possible eigenstates have equal probabilities.

The von Neumann entropy is mathematically equivalent to the Gibbs entropy, with the $\eta_{i}$ replaced by $\mathrm{p}_{\mathrm{i}}$, denoting the probabilities of classical states, and to the Shannon information entropy, with $P_{i}$ denoting the probabilities of events. These entropies are all subjective measures of an observer's uncertainty. They are not fundamental properties of state within the HCM framework.

The concept of refinement was introduced by Robert Griffiths in his Consistent Histories Interpretation of quantum mechanics [13]. Refinement results from a change in measurement framework when a single projector (potential measurement) is replaced with multiple consistent measurement possibilities. More generally, the entropy of refinement reflects the creation of potential microstates, caused by a change in a contextual parameter. A falling ambient temperature, for example, results in an increased resolution (refinement) of the system's configuration space, and a higher number of potential microstates. As a system adjusts to its changed context, it randomly selects and actualizes a newly created potentiality or sequence of potentialities.

The entropy of refinement is mathematically equivalent to the von Neumann entropy (10), where the probabilities express the uncertainty in the actualization of potentialities. The actualization of potentialities is intrinsically random, and the entropy of refinement is therefore an objective contextual property of state within the DCM framework. Expressed graphically, the entropy of refinement is a measure of the branches and probabilities in a system's forward trajectory.

Equation 9 describes the increase in entropy of refinement associated with a decline in the ambient temperature. But non-thermal changes can also result in refinement. To illustrate 
non-thermal refinement, we can consider a polarized photon. The photon's context is defined by its measurement set-up, which includes a polarizing filter and a photographic plate to record the photon if it passes through the polarizer.

When the polarized photon is initially generated, the polarized filter is parallel to the photon source's polarizer. There is only a single potentiality: the photon will pass unimpeded through the polarizer and it will be deterministically detected and recorded. The photon's trajectory is definite, and its entropy of refinement is zero.

If we rotate the detector's polarizer 45 degrees while the photon is in mid-flight, two measurable potentialities are created: the photon might pass through the detector's filter and be detected, as before, or it might be absorbed by the filter. The probabilities for each are $50 \%$, and from (10), the photon's entropy is $\log (2)$. The photon's contextual entropy increases due to rotation of its detector and change in its context.

When the photon does interact with the detector, it is either absorbed by the filter, or it passes through and is recorded by the photographic plate. Whether or not we observe or know the result, the photon's path and final state are definite and measurable. After interaction, the path's objective uncertainty, and consequently the photon's entropy of refinement, are zero.

The overall measurement process is irreversible, and it therefore produces entropy. It first produces entropy of refinement when the polarizing filter is rotated away from parallel to the photon's source. It produces entropy again when the photon's energy is irreversibly absorbed by the filter or recorded by photographic plate.

The changes in entropy of refinement described above are in marked contrast to HCM entropy. The HCM entropy is non-contextual, so it is unaffected by a mid-flight rotation of the analyzer and its entropy remains zero. Whereas the DCM's positive entropy of refinement declines to zero when the photon interacts with the detector and randomly chooses a definite trajectory and measurable state, the HCM describes an increase in the von Neumann entropy due to collapse of the zero-entropy superposed wavefunction to a mixed state.

The intrinsic randomness of quantum transitions is key to Schrödinger's cat thought experiment. The radioisotope in his experiment exists as a metastable state. It can momentarily exist in spontaneous transition between measurable states, but at no time does the particle or cat exist as part of a physically superposed state. A superposed state is not reversibly measurable, and it does not exist as a state within the DCM framework. There is only the spontaneous and random transition of a physical state to a new physical state of higher stability. The collapse of a wavefunction to a mixed state simply reflects the need for new information to account for the random changes in the physical state. Without that information, the wavefunction is incomplete and it describes a statistically mixed state. Observation and wavefunction collapse affect only the description and knowledge of the physical state-not the physical state itself. The measurement problem thereby vanishes.

\section{The Dissipative Conceptual Model of Time}

\subsection{The Two Components of System Time}

The DCM recognizes two fundamental and distinct components of time. Mechanical time describes the reversible and deterministic change of a system during intervals between transitions, while it exists as a state. Thermodynamic time describes the production of entropy during an irreversible process of transition from a metastable state to a higher-stability state.

Mechanical time in relativity is defined by a coordinate on the time axis in $4 \mathrm{D}$ spacetime. Mechanical time is conventionally defined as a real-valued coordinate, but this is merely a matter of convention. The DCM adopts a different convention, by replacing the notation for real-valued time $t$ with the mathematically equal $-i\left(i t_{m}\right)$, where $i$ is the square root of negative one and $i t_{m}$ is an imaginary coordinate for mechanical time. The DCM changes mechanical time to an imaginary parameter, but it leaves all equations of mechanics unchanged. The DCM expresses the 
time-dependent quantum wavefunction for an isolated and metastable (non-reactive) quantum system, for example, by:

$$
\psi\left(x, i t_{m}\right)=e^{\frac{-i t_{m} E}{\hbar}} \psi_{o}(x) .
$$

Except for the change in the function's argument for time, equation 11 is identical to the conventional expression for the system's time-dependent wavefunction.

Thermodynamic time records a system's irreversible production of entropy due either to the dissipation of exergy or to refinement. The decline in exergy for a first-order kinetic system ${ }^{3}$ is given by:

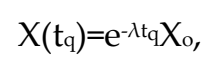

where $X_{0}$ is the initial exergy, $\lambda$ is a dissipation rate constant, and $t_{q}$ is the real-valued thermodynamic component of system time. Equation 12 describes, for example, the dissipation of exergy during radioactive decay. At time zero, the system's exergy equals its initial exergy, $X_{o}$, and as time advances toward infinity, the system approaches zero exergy at equilibrium. Thermodynamic time depends contextually on the ambient temperature, and it is therefore incompatible with, and ignored by, the HCM and conventional interpretations.

System Time: Equation 12 describes the continuous dissipation for a many-particle thermodynamic system. In the quantum limit, dissipation by an unstable positive-exergy particle is discontinuous. Periods with no dissipation mark intervals during which the particle exists as a well-defined and reversibly measurable metastable state. Its time evolution is indexed to a reversible time coordinate. At some point, however, the particle irreversibly transitions to a more stable state. An irreversible transition marks an interval of entropy production and an irreversible advance in thermodynamic time. A metastable quantum particle therefore requires both mechanical time and thermodynamic time to describe its behavior.

The DCM recognizes system time as a complex property of state, comprising both real-valued thermodynamic time and imaginary mechanical time. System time is represented by a point on the complex system-time plane (Figure 3A). A change over imaginary mechanical time (vertical axis) conserves exergy and describes the reversible and deterministic changes between irreversible transitions or measurements, within a single instant of irreversible thermodynamic time. A change over real thermodynamic time (horizontal axis) describes an irreversible transition to a more stable state and the production of entropy.
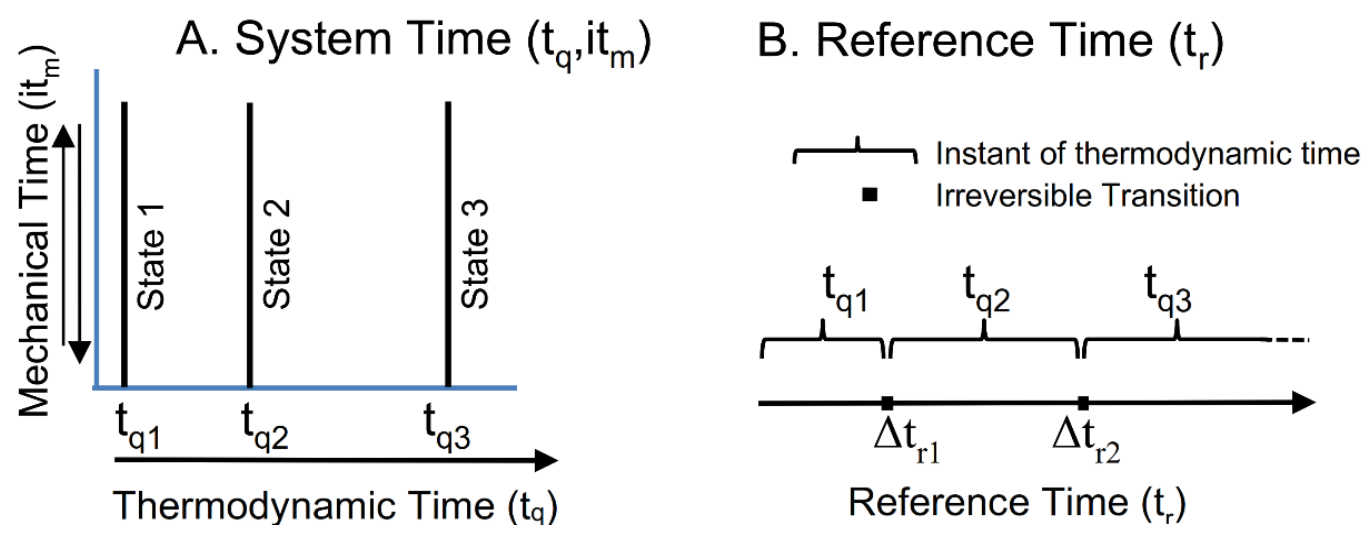

Figure 3. Complex System Time and Reference Time. Figure 3A shows the complex system-time plane, spanned by real-valued thermodynamic time (horizontal axis) and imaginary mechanical time (vertical axis)

Figure $3 \mathrm{~B}$ shows the irreversible advance in an observers' reference time during changes in

\footnotetext{
${ }^{3}$ One in which the system's rate of exergy dissipation is proportional to its exergy at fixed ambient temperature
} 
system-time. $\Delta \operatorname{tr}_{1} \& 2$ are advances in reference time during irreversible transitions. The intervals between transitions mark passage of reference time during reversible changes in mechanical time.

\subsection{Reference Time}

System time, whether it proceeds reversibly or irreversibly, is empirically measured by the advance of reference time, $t_{r}$, as recorded by an observer's reference clock (Figure 3B). Counting oscillations and recording memories is an inherently irreversible process [25]. The DCM provides a decoupling between system time and reference time. Reference time marks the continuous and irreversible "flow" of time, and it provides the empirical time scale across which events are recorded.

\subsection{Space, Time, and Nonlocality}

We now offer an explanation of how quantum nonlocality coexists with relativity. We first consider simultaneous measurements of entangled photons at points A and B by Alice and Bob (Figure 4), using parallel vertically polarized filters. The entangled electron pair has zero spin and no net polarization. Experiments show that if Bob measures a vertically polarized photon, then Alice measures a horizontally polarized photon, even when measurements are simultaneous. Individual measurement results are random, but they are strictly and instantaneously correlated.

The shaded light cones show the allowable domain for causal effects emanating from events at $\mathrm{A}$ and $\mathrm{B}$ within the empirical constraints of relativity and locality. The instantaneous correlation of physically separated measurements at points A and B, outside of each other's light cone, graphically illustrates the nonlocality of the photon pair's correlated measurements. Einstein famously referred to nonlocal correlations as "spooky" action at a distance.

The correlated results using parallel polarizing filters allow a simple interpretation, without invoking nonlocal superluminal interactions. As suggested by Einstein, Podolsky, and Rosen [21], measurement of one photon might simply reveal a preexisting but "hidden" property of the entangled state. Hidden properties could be inherited from the photon pair's common origin, and they could determine the measurement outcomes, which only appear random. In this case, discovering this property simply reveals the determined outcome of the other photon's measurement.

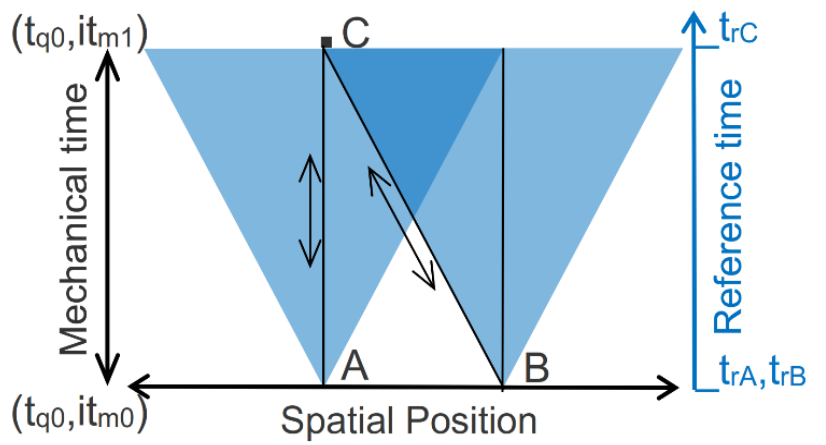

Figure 4. Instantaneous Correlation of Spatially Separated Measurements. The figure spans space (projected onto the horizontal axis) and mechanical time (left vertical axis) at a single instant of thermodynamic system time at $\mathrm{t}_{\mathrm{q} 0}$. Alice and Bob simultaneously record their measurement results for the photons at points A and B.

Superimposed on the diagram are two light cones, bounded by spherical light pulses from points A and $\mathrm{B}$ advancing across reference time (right vertical axis). Alice records her results at reference time $t_{r A}$ and at subsequent reference time $\operatorname{trc}_{r}($ point $C$ ), she receives the results Bob recorded at point $B$.

However, in 1964, John Bell devised a statistical test for preexisting local variables using non-parallel analyzers [18]. Numerous experiments have demonstrated that the statistics of multiple measurements violate Bell's test [26]. They prove that preexisting properties determining the photons' polarization or measurement results cannot exist, unless those properties are themselves 
nonlocal. If we assert realism, meaning that quantum properties exist independent of observation or observers, then we have to accept that quantum nonlocality and relativity coexist.

The DCM explains the coexistence of quantum nonlocality and relativity by recognizing system time as comprising both thermodynamic time and mechanical time. A system's change over mechanical time proceeds with no entropy production. No change in entropy means that forward and backward directions are equally probable. Mechanical time is not just reversible; it is also time symmetrical. With time symmetry, there is no distinction between forward and backward directions and there is no arrow of mechanical time. Asserting that an initial state causes a future state and asserting that a future state causes the initial state are equally valid. This expresses the idea of retrocausality $[27,28]$, and it exists over time-symmetrical mechanical time.

The photon measurements involve random transitions to states with definite (and correlated) polarizations. At point B, Bob reversibly records his result and transmits it to Alice via a signal photon polarized with the orientation that he measured. Alice reversibly records her photon's measurement result, and based on her results, she knows the orientation of Bob's entangled photon and his signal photon. The light cone and Bob's signal photon both reach Alice at point C. Knowing the signal photon's orientation, Alice reversibly measures it and confirms the correlation of their results.

Alice's observation of her measurement at A, Bob's observation and transmission of his measurement at $B$, and Alice's measurement of Bob's signal photon at $C$ are all reversibly conducted over mechanical time. The time-symmetry of mechanical time links the spatially separated measurement results by a deterministic chain of causality and retrocausality: $A \leftrightarrow C \leftrightarrow B$. No hidden variables or spooky action is required to explain the deterministic and nonlocal correlation of measurements at $\mathrm{A}$ and $\mathrm{B}$.

We next consider measurement using obliquely oriented polarizers. If Bob's analyzer is rotated 45 degrees, the system's contextual framework changes. Bob's and Alice's measurements are no longer strictly correlated. Alice can no longer know Bob's result based on her own results, and she cannot reversibly measure his signal photon. The time-symmetry link of causality and retrocausality connecting their measurement results is broken. The observed statistics of measurement results with obliquely oriented polarizers, however, are readily explained as random and local measurements, given only the photon pair's net-zero spin inherited from its source. Again, no hidden variables or spooky action is required to explain the observed results. The DCM successfully explains the mechanistic details of how relativity and quantum nonlocality compatibly coexist.

The righthand axis of Figure 4 shows the record of Alice's measurement events at points $\mathrm{A}$ and $C$, as measured by her reference clock. Even though the events at A, B and C are correlated within an instant of thermodynamic time, Alice experiences the irreversible passage of time between recording her measurement at time $t_{r A}$ and Bob's measurement at $t_{r}$. The irreversible flow of an observer's reference time and the empirical constraints of relativity preclude superluminal exchange of information between observers across their reference time. This explains the empirical compatibility between nonlocality and relativity.

\section{The Dissipative Conceptual Model of Process}

Up to this point, we have focused on states. The Second Law (and Postulate 4) describes the relative stabilities of states and the potential for a metastable state to transition irreversibly to a state of higher stability. It says nothing about the irreversible process of transition within non-equilibrium systems. Non-equilibrium thermodynamics is "a work in progress, not an established edifice" [29]. The conceptual problems of defining entropy in terms of fundamental physical principles for non-equilibrium systems are further discussed in [30] and [31]. The DCM, however, does provide a logically consistent framework that extends classical thermodynamics from the study of equilibrium systems, in which nothing every really happens, to nonequilibrium systems, in which exergy gradients can drive interconnected networks of energy and component flows and the self-organization of dissipative structures [32]. Within the framework of DCM, SDCM, ambient heat, and exergy are well-defined properties for non-equilibrium systems and contextual generalizations 
of entropy $\left(S_{T D}=\int_{0}^{T_{S y s}} \frac{d q}{T}\right)$, equilibrium heat $\left(\mathrm{T} \times \mathrm{S}_{\mathrm{TD}}\right)$, and Free Energy $(\mathrm{E}-\mathrm{TS} \mathrm{TD})$. In this section, we shift from the physical description of states to the DCM description of non-equilibrium systems and their dissipative processes.

\subsection{The Dissipative Homeostate}

Figure 5 extends the dissipative dynamics model for states (Figure 1) to a stationary dissipative system. The dissipative model assumes a stationary environment, but it is nonequilibrium. The system's ambient surroundings includes one or more sources of exergy or high-exergy material components. A system with stationary exergy sources and environment will converge over time to a stationary process of dissipation. The system is stationary, but it is not microscopically static, and it is not an actual state, as its constituents are constantly reorganizing and dissipating exergy. We refer to a stationary dissipative system as a homeostate. A homeostate is a generalization of David Duetsch's constructor [33,34], reformulated within the DCM framework.

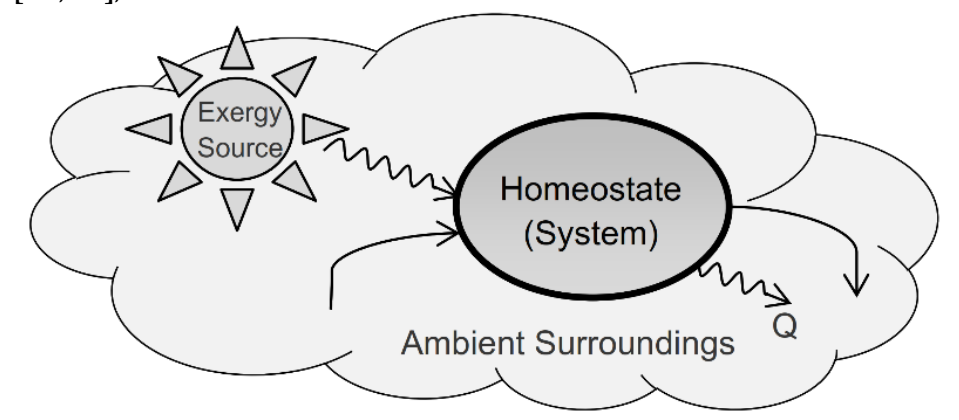

Figure 5. Homeostate Model. The system's nonequilibrium surroundings includes exergy source(s), either directly (e.g. sunlight) or indirectly, by high-exergy components. Ambient resources in the surroundings are also freely available to the system for processing and discharge. At homeostasis, time-averaged inputs of materials and energy equal outputs.

For a near-equilibrium system, energy flow is proportional to gradients. Fourier's Law, Fick's Law and Ohm's Law express the linearity of fluxes and gradients in potential for heat conduction, chemical diffusion, and electrical flow, respectively. Linearity defines the near-equilibrium regime. A near-equilibrium system converges to a unique steady-state dissipative process consistent with its steady-state boundary constraints and conservation laws.

Far from equilibrium, linearity breaks down. At a critical temperature gradient, for example, heat flow dramatically increases as a fluid spontaneously reorganizes itself from conduction to convection. Non-linearity can allow multiple dissipative solutions and multiple homeostates, all consistent with the system's boundary constraints and conservation laws.

We can express a homeostate as a network of links, pods, and dissipative nodes. Figure 6 shows the dissipative network for the Brusselator reaction [32]. The Brusselator is a theoretical model for an autocatalytic chemical reaction exhibiting oscillations. It has two sources, one for component 1 in state A and one for component 2 in state B. Links are the pathways for energy and components to flow from external sources, through the system, and back to the surroundings. Pods provide transient storage capacity for energy and components to accommodate fluctuations in flow rates. Nodes represent irreversible transitions. All dissipation within the dissipative system is assigned to nodes. Elementary nodes and transitions are contextually defined by perfect measurement at the ambient temperature, and they have no internal details. 


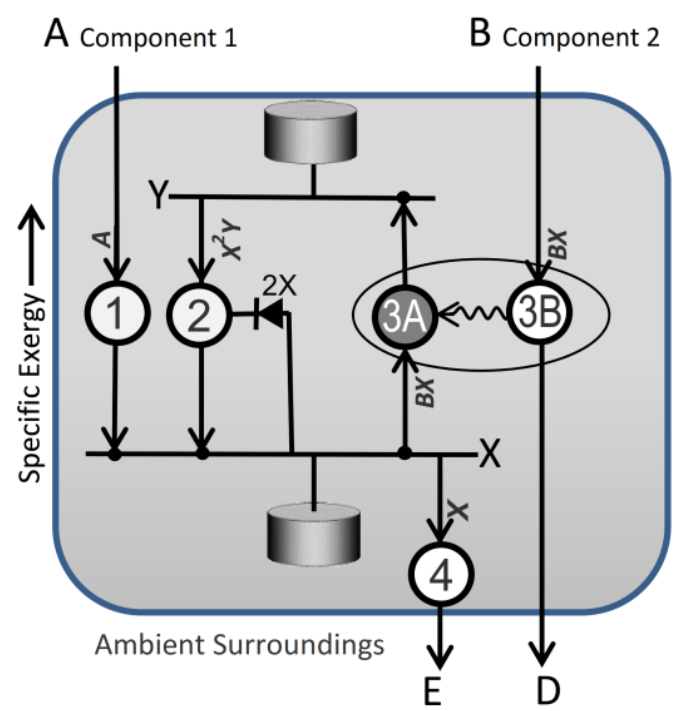

Figure 6. The Brusselator Network Model. Letters refer to the components' states. States higher on the diagram have higher specific exergy. The Brusselator comprises four reaction steps:

R1: External source $\mathrm{A} \rightarrow \mathrm{X}$

$\mathrm{R} 2: \mathrm{Y}+2 \mathrm{X} \rightarrow 3 \mathrm{X}$

R3: External source $B+X \rightarrow Y+D$ (discharge)

$\mathrm{R} 4: \mathrm{X} \rightarrow \mathrm{E}$ (discharge)

The Brusselator has four reactions, R1 to R4, listed in the Figure 6 caption. R1, R2, and R4 are exergonic transitions of component 1 . An exergonic transition is a transition of a component from a state of higher specific exergy to a state of lower exergy. Reaction 3 is a coupled reaction, involving two separate transitions. It is represented by nodes 3A and 3B. Node 3B is another exergonic transition, dissipating component 2 from state $B$ to state $\mathrm{D}$. The component's exergy is only partially dissipated, however. Some of the exergy is transferred to node $3 \mathrm{~A}$ via an exergy link (wavy arrow). Node $3 \mathrm{~A}$ is an endergonic transition. An endergonic node utilizes an exergy supply to lift a component "uphill" to a higher-exergy state. Node 3A lifts component 1 from state $\mathrm{X}$ to state $\mathrm{Y}$.

Transition rates, based on simple reaction rate theory, are shown by the arrowhead expressions in the figure. The kinetic rate coefficient for the forward direction is set to unity, and the reverse direction is assumed to be much slower and is set to zero. So, for example, the transition rate for R3 $(\mathrm{B}+\mathrm{X} \rightarrow \mathrm{D}+\mathrm{Y})$ is simply $\mathrm{BX}$. Reaction 2 is an autocatalytic transition of component 1 from state $\mathrm{Y}$ to state $X$. Autocatalysis means that the product partakes in the reaction. For reaction $R 2(Y+2 X \rightarrow 3 X)$, the transition rate is $X^{2} Y$, making the Brusselator non-linear.

Nodes represent transitions of components from one state to another. Numbers refer to the reactions listed above. Reaction $R 3$ couples two separate component transitions: $X \rightarrow Y$ and $B \rightarrow D$. The transition for node 2 is autocatalytic, meaning that the product partakes in the reaction. Expressions at the arrowheads denote component flow rates through the nodes. The appended pods represent the links' storage volumes. Pods allow non steady-state components flows. 


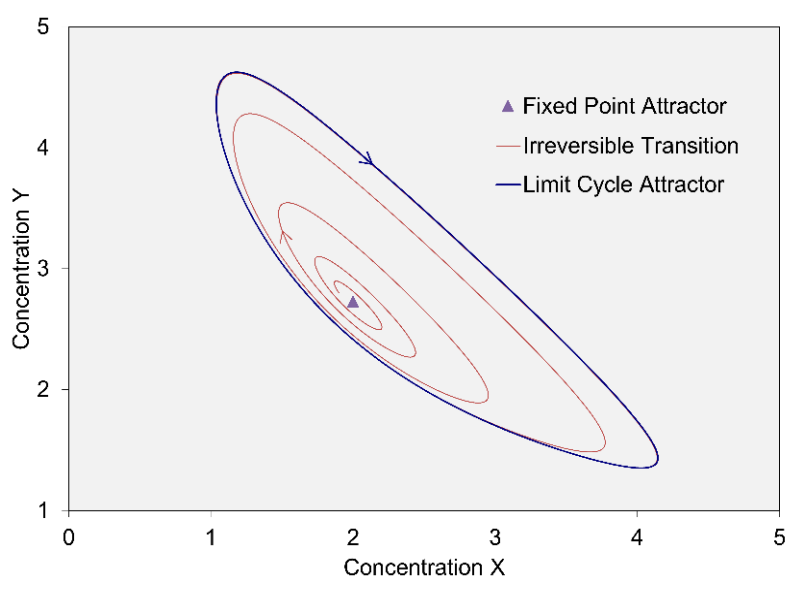

Figure 7. Brusselator Homeostates.

Setting the net production rates of $X$ and $Y$ to zero yields the steady-state concentrations $X=A$ and $\mathrm{Y}=\mathrm{B} / \mathrm{A}$. For a sufficiently high value of $\mathrm{A}$, the steady state homeostate is unstable to perturbations.

Any perturbation from the steady state homeostate sends the system on a transient path that converges to a stationary periodic homeostate, in which the concentrations of $\mathrm{X}$ and $\mathrm{Y}$ cycle. Steady-state and periodic homeostates are graphically illustrated in Figure 7 as attractors [35]. The steady state homeostate is represented by the fixed-point attractor and the oscillating homeostate is represented by the limit cycle attractor. A homeostate can also be dynamically chaotic, represented by a strange attractor. In all cases, an attractor is fixed, and it represents a stationary homeostate.

\subsection{The Constructive Power of Dissipation}

Dissipation can be much more than the dissipation of exergy into waste heat; it is the driver for all change, constructive as well as destructive. Nature is replete with examples in which systems become increasingly organized and evolve toward higher organization [32]. These changes occur within far-from-equilibrium systems that are sustained by the throughput and dissipation of external sources of exergy.

Lord Kelvin recognized the constructive power of dissipation in an article he wrote in 1862 [36]. He began by describing heat death, when all directed activity ceases, as the inevitable end-result of dissipation within a finite universe. He then proceeded to express a much deeper and overlooked idea. Backing off on the inevitability of heat death, he continued that the universe is in a state of "endless progress...involving the transformation of potential energy into palpable motion and hence into heat." In essence, he asserted that a system tends to defer dissipation by first utilizing exergy for palpable work, before eventually dissipating it into heat.

When Lord Kelvin stated this idea, classical mechanics was well entrenched in physical thought. Kelvin's idea was incompatible with classical mechanics, so it never gained a foothold and was largely ignored. His idea is fully compatible with the DCM, however, and we formalize his insight with Postulate 5:

Postulate 5 (Kelvin Selection Principle): Of the multiple paths by which a system can take exergy from a source and dissipate it to the surroundings, the path that maximizes the utilization of exergy is the most stable.

We formally define a homeostate's utilization $U$ as the sum of time-averaged internal work rates $\langle\dot{W}\rangle$ over its endergonic nodes:

$$
U=\sum_{i \in\left\{\begin{array}{c}
\text { ender- } \\
\text { gonic } \\
\text { nodes }
\end{array}\right\}}\left\langle\dot{W}_{i}\right\rangle,
$$


where $\dot{W}=\Delta \bar{X}_{i} \times J_{i}, \Delta \bar{X}_{i}$ is the increase in the specific exergy (exergy per unit of component), $\mathrm{J}_{\mathrm{i}}$ is the rate of flow of component through node $i$, and the angle brackets denote time-averaged values. As with exergy, the rate of internal work is measurable from, and defined with respect to, the ambient surroundings.

Postulate 5 states that the available pathway with the highest rate of internal work is the most stable. The Kelvin Selection Principle (KSP) is analogous to the Second Law of thermodynamics, but whereas the Second Law describes the selection and relative stability of states in terms of entropy, the KSP describes the selection and relative stabilities of dissipative processes in terms of exergy utilization.

A simple real-world illustration of the KSP is the stability of convection over conduction. Heat added to a convecting liquid does work of thermal expansion of the fluid to maintain density gradients. This is internal work necessary to sustain convective flow. Heat added to a static fluid, in contrast, is completely dissipated by conductive heat flow, without doing any work. The KSP therefore says that if boundary conditions and non-linearity allow both convection and conduction, convection is the more stable homeostate. Observations invariably show that this is the case.

A more revealing illustration of the KSP is the Miller-Urey experiment. Stanley Miller and Harold Urey took a mixture of gases, which they believed represented Earth's primordial atmosphere, and they stimulated it with electrical sparks to simulate lightning [37]. When they analyzed the gas mixture afterward, they found that the gas molecules had rearranged themselves into a variety of amino acids. The gas mixture started in a low-exergy near-equilibrium state and it ended up in a high-exergy far-from-equilibrium state. The sparks added exergy to the gas mixture, but instead of irreversibly dissipating the exergy directly to heat, the gas mixture deferred dissipation and utilized it to do work of creating high-exergy amino acids.

In the case of convection, perturbation analysis shows that, given a heat source and an unstable temperature gradient, a random perturbation will send the fluid down any one of many deterministic paths, all leading to convection. Starting with an equilibrium mixture of gases, however, randomly selecting a pathway that deterministically leads to amino acids would seem extraordinarily unlikely. Yet, the Miller-Urey experiment is repeatable with similar results each time. The KSP offers an alternate explanation, in which amino acid synthesis occurs through a sequence of incremental steps. Each step selects from multiple possibilities, not based on random selection, but instead guided by the preferential selection of higher-utilization pathways. The end-result of successive increments of internal work is the creation of high-exergy amino acids.

The DCM recognizes two common paths toward higher utilization: 1) increasing the net rate of exergy extraction (Rxx) from the surroundings and 2) increasing functional complexity. Rxx is given by:

$$
\mathrm{R}_{X X}=\langle\dot{\mathrm{X}}\rangle_{\text {input }}-\langle\dot{\mathrm{X}}\rangle_{\text {output }}=\sum_{\mathrm{i} \in\{\text { inputs }\}}\left\langle\overline{\mathrm{X}}_{\mathrm{i}} \mathrm{J}_{\mathrm{i}}\right\rangle-\sum_{\mathrm{i} \in\{\text { outputs }\}}\left\langle\overline{\mathrm{X}}_{\mathrm{i}} \mathrm{J}_{\mathrm{i}}\right\rangle
$$

where $\dot{\mathrm{X}}$ and $\mathrm{J}_{\mathrm{i}}$ are the rates of exergy and component flows into or out of the system and $\overline{\mathrm{X}}$ is the specific exergy. Perhaps the simplest way to increase $\mathrm{Rxx}$ is by expanding or replicating a dissipative process, given an expandable exergy source. This would proportionately increase both the exergy extraction rate and its internal work rate (utilization). Given sufficient resources, this path drives a species to expand its population, thereby increasing its net Rxx.

The second path toward higher utilization is increasing functional complexity. The term complexity is commonly associated with the amount of information required to specify a system's state. We are not interested here in the complexity of a state, however. Rather, we are interested in the complexity of a system's dissipative process. If, as proposed by Lord Kelvin, a system defers dissipation by doing palpable work on some other dissipative system, then that system could likewise defer dissipation. The recursive deferral of dissipation to sustain other dissipative systems leads to an expanding network of agent-nodes of increasing interconnectedness and organization. This idea precisely expresses the concept of functional complexity.

We formally define functional complexity as the ratio of utilization (equation 13) to net rate of exergy extraction (equation 14): 


$$
\mathrm{C}_{\mathrm{F}}=\frac{\langle\mathrm{U}\rangle}{\mathrm{R}_{X X}} .
$$

Functional complexity is a measurable and well-defined property of a homeostate's dissipative process. For a single pass of exergy and a single endergonic node, functional complexity can approach unity for perfect efficiency and no dissipation. However, a homeostate can increase its functional complexity and utilization well beyond unity by reprocessing and recycling exergy via feedback loops (Figure 8) or by sustaining a network of endergonic nodes. Feedback loops are ubiquitous within biological systems, from cells to ecosystems, leading to higher functional complexity.

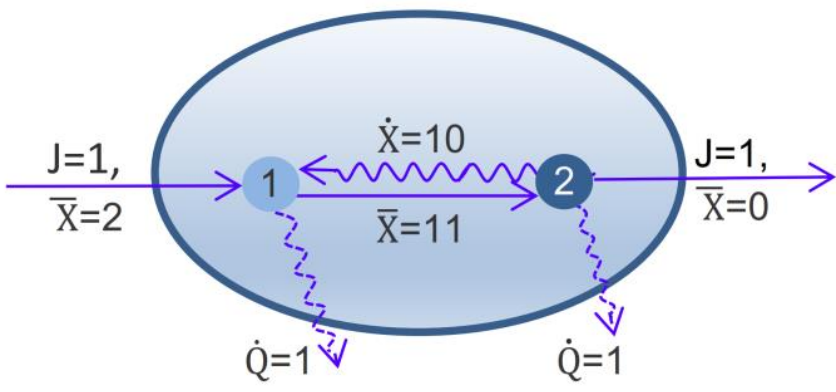

Figure 8. Simple Feedback Loop. A component flows through system (straight vectors) at a rate (J) of one unit. Its specific exergy $(\overline{\mathrm{X}})$ equals two units at input and zero at output. The rates of net exergy input $(\mathrm{J} \times \overline{\mathrm{X}})$ and total dissipation rate $(\dot{Q})$ equal two units. Mass and energy inputs and outputs are balanced.

Exergonic node 2 takes the component with 11 units of specific exergy, dissipates one unit, and supplies exergy to endergonic node 1 (wavy vector) at a rate of 10 units. Endergonic node 1 uses this exergy to do 10 units of internal work to lift the specific exergy of component from 2 units to 12 units. Node 1 dissipates one unit of exergy and outputs the component with 11 units. The system's internal work rate equals 10 units. With 2 units of exergy input, its functional complexity $\mathrm{C}_{\mathrm{F}}$ equals 5 .

The Brusselator's steady-state and cycling homeostates have virtually identical rates of dissipation and net exergy extraction rate. They differ, however, in their internal work. For the steady state homeostate, the concentrations of $X$ and $Y$ are fixed. There is never a measurable transfer of component 1 "uphill" from state $\mathrm{X}$ to $\mathrm{Y}$, and endergonic node $3 \mathrm{~A}$ does no internal work on the system. For the oscillating mode, however, component 1 periodically accumulates in and is released from the $X$ and $Y$ pods. During net transfer from $X$ to $Y$, endergonic node 3B does internal work of lifting component 1 to higher exergy. The oscillating homeostate has higher internal work rate and higher utilization than the steady-state mode. The KSP asserts that the oscillating mode is more stable, in agreement with perturbation analysis (Figure 7). With a fixed Rxx, equation (15) shows that the oscillating homeostate also has higher functional complexity.

We can generalize this conclusion and assert that an oscillating homeostate is more stable than a steady state homeostate, other differences being negligible. The spontaneous emergence of resonance, commonly observed in mechanical or fluid mechanical systems, illustrates spontaneous cycling and the arrow of increasing functional complexity.

Systems of linked oscillators are often observed to synchronize in a process known as entrainment. Christiaan Huygens, the inventor of the pendulum clock, first documented this in 1666, when two of his pendulum clocks mounted on a common support spontaneously synchronized [38]. Exergy gradients between pairs of linked, but unsynchronized oscillators, will dissipate exergy and subtract from the system's utilization. If oscillators are synchronized, however, there are no exergy gradients between oscillators and no associated dissipation, so utilization increases. The KSP provides a general principle that explains both oscillations and their synchronization, independent of a system's specific mechanics. The KSP can explain the development of oscillations and synchronization, which is prevalent throughout the universe, at all scales [39]. 
As a final illustration of the KSP and the arrow of functional complexity, we consider whirlpools. We commonly observe whirlpools, indicating they are often more stable than the less organized and lower-complexity radial flow of water directly toward a drain. We considered a simple model for a draining cylinder of water maintained at steady state. The whirlpool model's internal work rate of accelerating water through a vortex was 4,000 times higher than for the radial flow model's rate of internal work. The stability of the whirlpool reflects its higher internal work rate (utilization). At the same time, the whirlpool's rate of exergy extraction is lower than for radial flow. The centrifugal force of a whirlpool's circulation lowers the water level and pressure over the drain. This actually reduces the whirlpool's steady-state rate of water throughput and its rate of exergy supply. The whirlpool's lower Rxx increases the whirlpool's functional complexity to 5,000 times higher than radial flow.

The stability of the whirlpool, with it lower rate of dissipation and entropy production, provides an important counterexample to the idea that "faster is better." It falsifies the maximum entropy production principle [40-42], which asserts that the rate of exergy dissipation and entropy production always tends to be maximized.

\section{Discussion}

\subsection{Why DCM?}

The DCM and non-contextual Hamiltonian interpretations present alternative models of physical reality. They differ only in their definitions of perfect measurement, their assumptions, and their interpretations of empirical facts. Any viable interpretation must be fully consistent with empirical measurements. However, while consistency is essential, a model's assumptions should be empirically justifiable and its implications should be testable.

If we assert a physical reality that is independent of its ambient context and we define perfect measurement in the absence of thermal noise, then empirical observations imply eternalism, in which the universe unfolds deterministically and the future, as well as the past, is set in stone [43,44]. It means we either need to accept an exquisitely tuned and physically unexplained initial state of the universe to account for its current state of organization [45] or accept that we are a statistical outlier within a multiverse of universes [6]. And, we must choose among 1) the potential existence of superposed cats or an unexplained mechanism of physical collapse within an isolated (and unobserved) system; 2) an exponentially growing multiplicity of branching universes, in which everything that can happen does happen in some branch [15], or 3) a subjective reality defined by an individual's experience of it [14]. These and other interpretations of quantum mechanics are consistent with empirical observations, but their assumptions are not empirically justified and their metaphysical implications are not testable.

The DCM is based only on assumptions that are empirically justifiable. No system is perfectly isolated and real systems interact with their ambient surroundings at a positive absolute temperature. Consequently, the DCM defines a system as it exists within its actual context by perfect measurement from its ambient surroundings. The DCM's contextual framework with positive-temperature ambient surroundings provides objective and fundamental randomness of environmental fluctuations. This is the essential key required to fulfill Prigogine's ambition of establishing fundamental irreversibility and the end of certainty $[5,6]$.

By abandoning non-contextuality, the DCM can explain nonlocal correlations of measurements without violating relativity. It can explain the unfolding of the present from an indefinite future; it establishes irreversible dissipative process as a fundamental property of non-equilibrium systems, and it explains evolution toward higher functional complexity and stability, guided by the Kelvin Selection Principle. The DCM's explanations are retrodictions of well-established empirical facts and validation of its implications.

The DCM is an improvement over non-contextual interpretations of physics, but it is still just a model of physical reality. Definition 2 for perfect measurement is based on effectively infinite ambient surroundings, so that it is fixed during perfect measurement. The DCM models the state's 
ambient surroundings as equilibrium, defining a single unique ambient temperature. Reality is more complex, however. In a non-equilibrium universe, the surroundings are finite and not in equilibrium. Different components of a system might interact with different regions of the surroundings. A system might thermally interact with its adjacent surroundings, but electronic transitions might interact with energetic and non-equilibrium photons. A metastable state could interact with its environment over a spectrum of energies and temperatures. The DCM models a homeostate with respect to a stationary environment, but nothing is fixed for eternity. Exergy sources run out; the environment can become contaminated with waste heat and components; and expansion of the universe drives down its ambient cosmic microwave background temperature. The DCM is a key step forward in modeling physical reality, but like any model, the DCM is a simplification of reality.

\subsection{Is Contextuality Objective?}

The DCM defines states and homeostates within the context of its ambient surroundings, with which a system interacts or potentially interacts, and from which the DCM defines perfect measurement. But in a non-uniform universe, a system's surroundings depend on the system's delineation. We can delineate an experimental system from its cryogenic detectors; we can delineate the system and its measurement apparatus from its laboratory setting; or we can delineate the laboratory and the rest of the Earth from space. In each case, the system's ambient surroundings differs. The DCM simply accepts the delineation between a system and its surroundings as part of the system's contextual definition. Given a system's definition, the DCM's model of the physical state is independent of any particular ambient observer or the existence of observers. In this weak sense, at least, the DCM is objective.

We have argued that the cosmic microwave background defines an objective ambient temperature for the universe as a whole. The CMB defines an objective ambient temperature of 2.7 kelvin, permeating the vacuum of space. The CMB could provide a universal basis for an objective contextual interpretation.

The interpretation of relativity in terms of $4 \mathrm{D}$ spacetime is a consequence of assuming non-contextuality of spacetime and its independence of any particular inertial framework. However, Thyssen [46] has shown that 4D spacetime and 3D space with universal time are both consistent with the empirical data for Special Relativity. The DCM already recognizes the CMB as a contextual ambient reference. The $\mathrm{CMB}$ can also define an objective state of rest, for which any directional anisotropy in the CMB's doppler shift is zero (or at least minimized). To the extent that our focus is local and we can ignore the expansion of space, the CMB can locally specify an objective ambient temperature and a rest state for contextually defining 3D space with universal time. This expresses the concept of Presentism [2], and it is logically consistent with special relativity and with the contextuality of the DCM.

It is not clear whether the $\mathrm{CMB}$ can provide objective contexts for ambient temperature and rest state across an expanding universe. A universal ambient temperature across an expanding universe needs to be reconciled with Gavassino's argument that thermal equilibrium can be unambiguously defined only for bodies at relative rest [47]. An expanding universe violates the "no red shift" condition for defining Einstein synchronization [48]. This would seemingly preclude an expanding 3D space with universal time. Further investigation is required to determine whether it is possible to define objective and universal contexts for ambient temperature or for 3D space across an expanding universe.

\subsection{Emergence}

As the ambient temperature falls, energized metastable states emerge from previous states of equilibrium. Subatomic particles form as near-equilibrium ambient fields in extremely high-energy environments found in the early universe or in particle accelerators. As the ambient temperature falls, they are quenched as high-exergy field excitations, which we commonly describe as particles. As the ambient temperature continues to decline, new structures and organization can emerge at both microscopic and macroscopic scales. Ellis and Drossel clearly express these ideas [3]: 
"Channels for various types of reversible processes (particle physics, nuclear physics, atomic physics, molecular) close off as the universe evolves due to the cosmic temperature $\mathrm{T}(\mathrm{t})$ falling below the threshold for each of these processes, allowing the building up of metastable thermodynamic states as the temperature drops even further".

We can gain insight into emergence with declining ambient temperature by considering, once again, a classical ambient gas. A gas has a well-defined and non-contextual temperature, pressure, volume, and total energy. This defines the thermodynamic macrostate. The positions of the gas's particles, in contrast, are contextual properties defined in the context of the gas's ambient surroundings.

When the gas is in equilibrium with its ambient surroundings, it is in its ground-state microstate. The ambient gas's particles' positions, as determined by perfect ambient measurement, are completely defined by a probability distribution uniformly spread across the gas's configuration space.

While the gas temperature remains fixed and the ambient temperature falls, perfect ambient measurement and the system's configuration space become more refined. The probability distribution function for the particles' locations becomes increasingly particle-like. In the limit of absolute zero ambient temperature, each potential ambient state describes a distinct configuration of precisely defined positions and motions. This is the idealized statistical mechanical description of a gas.

As the ambient temperature falls, the gas particles also acquire exergy and a potential to interact irreversibly with the system's cooling ambient surroundings. Random inhomogeneities in the gas will lead to exergy gradients within the system. This, in turn, can lead to the emergence of macroscopically organized convective flows. The emergence of particles and their organization into macroscopic structures all occur in response to a declining ambient temperature.

\subsection{Cosmological Implications}

Ellis and Drossel promote the evolving block universe (EBU) model to resolve indeterminism and the origin of time's arrows [3]. Whereas the block model of the universe is a static block in 4D spacetime spanning all time since the Big Bang, the EBU is bounded at "now" by the unfolding of the present from an indeterminate future.

Ellis and Drossel attribute the indeterminism of "now" to quantum randomness. However, given that the wavefunction is a deterministic function, there is a conflict between two of Ellis's statements in a related article [49]:

1. "The view taken here is close to the Copenhagen interpretation: it assumes firstly that the wave function is real (i.e., descriptive and objective rather than epistemic)."

2. "Our basic view is that measurement is truly a stochastic, nonunitary process ... a 'wave function collapse.'”

The first sentence extends the determinism of the wavefunction to physical states. The second (correctly) acknowledges fundamental physical randomness associated with measurement, and this contradicts the first statement. Failure to explicitly reconcile this contradiction undermines EBU's logical consistency. However, Ellis and Drossel recognize a limitation of determinism [3]:

"when quantum effects are not dominant and suitable classical equations of state are given, as at times after the end of inflation, outcomes will be unique [i.e. determined]. However, this raises again the above-mentioned problem that deterministic equations require infinite precision"

They point out that determinism depends on infinite precision. Infinite precision is a consequence of HCM's definition of perfect measurement, in the idealized limit of zero thermal noise, i.e. at an absolute-zero ambient temperature. The DCM acknowledges the reality of a positive ambient temperature, and this leads directly to coarse graining of configuration space and to a breakdown of determinism. 
Coarse graining leads to indeterminate outcomes, but it does not establish the direction of indeterminate change or of time's arrow. In order to establish the direction of time's arrows, Ellis and Drossel invoke cosmic expansion and a past condition [3]:

"A global Direction of Time arises from the cosmological context of an expanding and evolving universe; This overall context, and particularly the associated decrease of temperature of interacting matter and radiation with time, together with a Past Condition of low initial entropy, leads to local Arrows of Time"

David Albert introduced the Past Hypothesis, stating: "We make the cosmological posit that the universe began in an extremely tiny section of its available phase space," i.e. in an initial state of low entropy [3]. This implies an extremely well-tuned past condition. The DCM has a different take on the past hypothesis. The universe started in a state of near-zero entropy, but it was an equilibrium ground-state microstate. As an equilibrium microstate, it filled the universe's initial configuration space, so it was the only initial state possible. No fine tuning was required.

Expansion and a declining ambient temperature subsequent to the Big Bang led to the refinement of the universe's configuration space and to the indeterminate unfolding of its future by random actualizations of newly refined and contextually defined potentialities. The universe has evolved from an initially hot state of near-equilibrium and zero-entropy, to its current state of high-exergy and high-entropy with respect to its cold ambient microwave background. Its positive exergy drives irreversible processes and the emergence of dissipative structures. As long as the universe expands and the ambient temperature continues to fall, it will continue to produce entropy and exergy, without ever reaching an equilibrium state of zero-exergy heat death.

\subsection{Origin and Evolution of Life}

The DCM sheds light on another perplexing question, on the origin of life. Once self-replicating autocatalytic systems exist, Darwinian natural selection can act to propagate beneficial variations and guide the evolution of life, but selection of the fittest cannot account for the origin of life or for evolution within non-replicating systems.

The Kelvin Selection Principle constitutes a fundamental law of evolution that applies to both replicating and non-replicating systems. The KSP can apply to a system of simple organic compounds that is open to exergy sources (e.g. Miller-Urey experiment), by progressively selecting dissipative processes and guiding the system toward higher functional complexity. By continually seeking to defer dissipation to do work on other agent-nodes, the KSP guides a system toward expanding networks of links and feedback loops to recycle exergy. Over time, self-organization can lead to autocatalytic and self-replicating networks. At that point, Darwinian evolution can take hold.

Once life is established, the KSP guides its evolution through an interplay between Darwinian competition to increase fitness and cooperation to sustain and increase functional complexity. Darwinian competition predominates when a species' dissipative cost of competition is small relative to the potential reward of increased exergy extraction rate. In this case, competition enables a species to achieve higher utilization by expanding its resource base. The KSP provides the drive for a species to expand up to the carrying capacity of its environment.

Cooperation predominates if resources are inelastic or the dissipative cost of competition is too high. When a rainforest's canopy completely covers the forest, for example, its solar resource base is inelastic to further gains. Over its fifty-million-year period of relative environmental stability and net exergy extraction rate, the Amazon rainforest has continued to evolve by developing highly complex webs of interaction for recycling components and exergy [50]. Ecological nutrient cycling [51] involves repeated utilization and renewal of the nutrients' specific exergy. From equation (15), this defines the system's functional complexity factor. By recursively deferring dissipation and recycling exergy, a dissipative system can increase its utilization by increasing its functional complexity, with no upper bound in the limit of perfect efficiency.

\section{Conclusions}


An interpretation is "good" if it is consistent with empirical observations, precise, parsimonious in its assumptions, explanatorily broad, falsifiable, and if it promotes scientific progress [52]. The Dissipative Conceptual Model and prevailing interpretations of physics all satisfy the consistency requirement. Prevailing interpretations define the physical state in isolation, devoid of context. But noncontextuality cannot possibly be justified by empirical observations, and parsimony argues against making unjustified assumptions. The Dissipative Conceptual Model is parsimonious by defining a system's physical state contextually, as it exists with respect to its ambient surroundings.

The DCM explains the thermodynamic arrow of time and the evolution of complexity as fundamental physical responses of non-equilibrium systems, without invoking an exceptional and unexplained initial state or improbable accident. It explains the empirical randomness of quantum measurements and the coexistence of nonlocality and relativity in terms of fundamental principles, without invoking empirically consistent but implausible and untestable metaphysical implications such as superposed cats, an exponentially branching universe, or spooky action at a distance. And, it extends the scope of physics from its traditional focus on states to irreversible dissipative processes, opening up new avenues of investigation within fields previously regarded as too high-level or too complex for fundamental physical analysis. By all of these measures, the DCM is a good interpretation of physical reality and a viable response to Ellis and Drossel's challenge [3]:

"the challenge is to find some alternative proposal [to the Evolving Bock Universe] that does justice to the fact that time does indeed pass at the macro scale, and hence it must be represented by something like the EBU structure presented here. To deny that time passes is to close one's mind to a vast body of evidence in a spectacular way."

Funding: This research received no external funding.

Conflicts of Interest: The author declares no conflict of interest.

\section{References}

1. Hawking, S., Penrose, R. The nature of Space and Time. Princeton University Press, Princeton, NJ USA 1996; 160pp

2. Rovelli, C. Neither Presentism nor Eternalism. Foundations of Physics, 2019, 49 (12). pp. 1325-1335 URL: http://philsci-archive.pitt.edu/id/eprint/16898 (accessed 2020-06-04).

3. Ellis, G. and Drossel, B. Emergence of Time arXiv 2019 arXiv:1911.04772v3

4. Skow, B. Objective Becoming. Oxford University Press, Oxford, UK. 2015; 249pp

5. Prigogine, I.; Stengers, I. The End of Certainty. The Free Press. 1997; 240pp ISBN 978-0-684-83705-5

6. Petrosky, T. and Prigogine. I.. Poincare resonances and the limits of trajectory dynamics. Proc. Natl. Acad. Sci. USA, 1993, 90, pp. 9393-9397 URL: https://www.pnas.org/content/pnas/90/20/9393.full.pdf (accessed 2020-08015)

7. Dodd, Matthew S.; Papineau, Dominic; Grenne, Tor; Slack, John F.; Rittner, Martin; Pirajno, Franco; O'Neil, Jonathan; Little, Crispin T. S. Evidence for early life in Earth's oldest hydrothermal vent precipitates (PDF). Nature 2017, 543 (7643), pp 60-64.

8. Kragh, H. Contemporary History of Cosmology and the Controversy over the Multiverse, Annals of Science, 2009 66:4, pp. 529-551, DOI: 10.1080/00033790903047725

9. Putnam, H. Time and Physical Geometry, Journal of Philosophy 1967, 64, pp. 240-247.

10. Rietdijk, C. W. A rigorous proof of determinism derived from the special theory of relativity, Philosophy of Science 1966, 33. pp. 341-344.

11. Kochen, S.; Specker, E. P. The problem of hidden variables in quantum mechanics. Journal of Mathematics and Mechanics. 196717 (1), pp. 59-87.

12. D'Ariano, G.M.; Paris, G.A.; Sacchi, M.F.; Quantum Tomography arXiv 2008, arXiv:quant-ph/0302028v1

13. Griffiths, R.B. The Consistent Histories Approach to Quantum Mechanics. The Stanford Encyclopedia of Philosophy (ed. Zalta, E.N.), https://plato.stanford.edu/entries/qm-consistent-histories/ (Spring 2017)

14. Caves, C. M.; Fuchs, C. A.; Schack, R. Quantum Probabilities as Bayesian Probabilities. Phys. Rev. A 2002 65(2). 
15. Vaidman, Lev. Many-Worlds Interpretation of Quantum Mechanics. The Stanford Encyclopedia of Philosophy (ed. Zalta, E.N.) https://plato.stanford.edu/archives/fall2016/entries/qm-manyworlds/ (Fall 2016)

16. Schlosshauer, Maximilian; Kofler, Johannes; Zeilinger, Anton. A Snapshot of Foundational Attitudes Toward Quantum Mechanics, Studies in History and Philosophy of Modern Physics, 2013, 44(3), 222-230, DOI: 10.1016

17. Faye, J. Copenhagen Interpretation of Quantum Mechanics. The Stanford Encyclopedia of Philosophy (ed. Zalta, E.N.), https://plato.stanford.edu/entries/qm-copenhagen/ (2019).

18. Bell, John S. On the Einstein-Podolsky-Rosen Paradox. Physics 1964, 1(3), pp. 195-200

19. Wikipedia: Bell Test Experiments. https://en.wikipedia.org/wiki/Bell test experiments. (Accessed on 15 Dec, 2018)

20. Goldstein, S. Bohmian Mechanics. The Stanford Encyclopedia of Philosophy (ed. Zalta, E.N.), https://plato.stanford.edu/archives/sum2017/entries/qm-bohm/ (Summer 2017).

21. Einstein, A.; Podolsky, B.; Rosen, N. Can Quantum-Mechanical Description of Physical Reality Be Considered Complete? Phys. Rev. 1935, 47, pp. 777-780

22. Mermin, D. Could Feynman Have Said This? Physics Today 2004, 57 (5), pp. 10-11

23. Wikipedia: Quantum Zeno effect. https://en.wikipedia.org/wiki/Quantum_Zeno_effect

24. Von Neumann, J. Mathematical Foundations of Quantum Mechanics. Princeton University Press. 1955. ISBN 978-0-691-02893-4.

25. Rovelli, C. Memory and Entropy. arXiv 2020, arXiv:2003.06687v1

26. Abellan, C. (and 101 others). Challenging local realism with human choices, Nature 2018. 557, pp. $212-216$. Available online arxiv:1805.04431v3 (accessed 15 Dec, 2018)

27. Leifer, M.S.; Pusey, M.F. Is a time symmetric interpretation of quantum theory possible without retrocausality? Proc. R. Soc. A 2017, 473, pp. 1-25. https://royalsocietypublishing.org/doi/full/10.1098/ rspa.2016.0607

28. Price, H. Does time-symmetry imply retrocausality? How the quantum world says "Maybe"? Studies in History and Philosophy of Science Part B: Studies in History and Philosophy of Modern Physics 2012, 43 (2), 75-83

29. Wikipedia: Non-equilibrium Thermodynamics https://en.wikipedia.org/wiki/Non-equilibrium thermodynamics

30. Lieb EH, Yngvason J. The entropy concept for non-equilibrium states. Proc R Soc A 2013469 (20130408). URL: http://dx.doi.org/10.1098/rspa.2013.0408 (accessed 2020-18-15)

31. Velasco, R.M.; Leopoldo Scherer Garcia-Colın, L.S.; Uribe, F.J. Entropy Production: Its Role in Non-Equilibrium Thermodynamics. Entropy, 2011 13, pp. 82-116; doi:10.3390/e13010082

32. Prigogine, I.; Nicolis, G. Self-Organization in Nonequilibrium Systems: From Dissipative Structures to Order through Fluctuations. Wiley Interscience, 1977, 512pp.

33. Deutsch, D. Constructor Theory Synthese 2013, 19018 p. 4331-59.

34. Deutsch, D.; Marletto, C. Constructor theory of information, Proceedings of the Royal Society A 2014, 471 (2174): 20140540. arXiv:1405.5563

35. Milnor, John. On the concept of attractor. Comm. Math. Phys. 1985, 99(2), pp. 177-195. https://projecteuclid.org/euclid.cmp/1103942677

36. Thomson, W. On the age of the sun's heat. Macmillan's Mag 1862, 5, p.288-93. https://zapatopi.net/kelvin/ papers/on the age of the suns heat.html (Accessed on 15 Dec, 2018)

37. Miller, S.L. Production of Amino Acids Under Possible Primitive Earth Conditions. Science 1953, 117 pp. 528-9

38. Oliveira, H.M. and Melo, L.V. Huygens synchronization of two clocks. Sci. Rep. 2015,5 (11548) https://www.nature.com/articles/srep11548

39. Strogatz, S. Sync: The Emerging Science of Spontaneous Order. Hyperion books, 2003338 pp. ISBN 0-7868-6844-9

40. Kleidon, A. and Lorenz, R. Entropy Production by Earth System Processes. In Non-equilibrium thermodynamics and the production of entropy - life, earth, and beyond; Springer-Verlag 2005; Kleidon, A. and Lorenz, Eds.; R. 260pp. ISBN 3-540-22495-5

41. Paltridge, G.W. Stumbling into the MEP Racket: An Historical Perspective. In Non-equilibrium thermodynamics and the production of entropy - life, earth, and beyond; Springer-Verlag 2005 Kleidon, A. and Lorenz, R.D. Eds.; 260pp. ISBN 3-540-22495-5 
42. Paltridge, G.W. A Story and a Recommendation about the Principle of Maximum Entropy Production. Entropy 2009, 11 (4) p. 945-948. https://www.mdpi.com/1099-4300/11/4/945

43. Winsberg, E. Can conditioning on the "past hypothesis" militate against the reversibility objections? Philosophy of Science 2004, 71 (4) pp. 489-504

44. Callender, C. Thermodynamic Asymmetry in Time. The Stanford Encyclopedia of Philosophy (ed. Zalta, E.N.) https://plato.stanford.edu/archives/win2016/entries/time-thermo/ (Winter 2016 Edition).

45. 't Hooft, G. Free Will in the Theory of Everything. arXiv 2017, arxiv:1709.02874v2

46. Thyssen, P. Conventionality and Reality, Found of Physics 2019, 49, pp.1336-1354

47. Gavassino, L. The zeroth law of thermodynamics in special relativity. arXiv 2020, arXiv:2005.06396v1

48. Macdonald, A. Clock synchronization, a universal light speed, and the terrestrial red-shift experiment, $A m$. Jour. of Phys., 1983, 51 (9) pp. 795-797, http://philsci-archive.pitt.edu/4863/1/Synch.pdf

49. Drossel, B; Ellis, G. Contextual Wavefunction Collapse: An integrated theory of quantum measurement arXiv 2018, arXiv:1807.08171v2

50. Burnham, R.J.; Johnson, K.R. South American palaeobotany and the origins of neotropical rainforests. Philosophical Transactions of the Royal Society 2004, 359 pp.1595-1610

51. Bormann, F. H.; Likens, G. E. Nutrient cycling. Science 1967, 155 pp. 424-429

52. Gieseler K., Loschelder D.D., Friese M. What Makes for a Good Theory? How to Evaluate a Theory Using the Strength Model of Self-Control as an Example. In Social Psychology in Action; Sassenberg K., Vliek M, Eds.; Springer, Cham 2019. 